\title{
ALGUNAS CONSIDERACIONES BIOGEOGRAFICAS SOBRE EL PACIFICO SUDORIENTAL
}

MANUEL VEGAS-VELE $Z$

Oficina Regional de Ciencia y Tecnologia de la UNESCO para América Latina y el Caribe

Montevideo, Uruguay

\section{SYNOPSIS}

The improved study of the Eastern South Pacific undertaken in the last years enables us to have a better view of the marine communities' biogeography. The author, elaborating on several ideas of previous studies, proposes four subdivisions or districts in that region: I) tropical of the Panamic Pacific province up to $\left.3^{\circ} 30^{\prime} \mathrm{S} ; 2\right)$ intermediate, from approximately $3^{\circ} 30^{\prime}$ to $70 \mathrm{~S}$, where tropical and southern waters in. fluence the presence of organisms; 3) temperate, of the Peruvian Coastal Current or Humboldt Current; and 4) tropical, in the waters off this last Current ioining the Peruvian Oceanic Current. The author, consulting extensive literature examines the presence of molluscs and fishes, in order to select a characterization of each biogeographical district. Some of the proposals are, for instance, Littorina peruviana, Ocypode guadichaudii and Engraulis ringens for the temperate district; Littorina paytensis, Olivella broggi and Scorpaena afuerae for the intermediate one; the thunids and Exocoetus volitans for the tropical oceanic waters; and Anadara tuberculosa, Littorina varia, Pomadasys panamensis and Sciades troschelli for the tropical waters north of $3^{\circ} 30^{\prime}$.

\section{El sistema de corrientes y las regiones biogeográficas}

Como sabemos, Humboldt en 1801 percibió la existencia de una corriente oceânica que recorría las costas del Perú y provenía, según interpretación del sabio, del Polo Sur. Esta corriente, que recibió luego el nombre de Corriente de Humboldt, tiene aguas relativamente frías para la latitud correspondiente y determina un clima muy particular para la costa Norte de Chile y la mayor parte del litoral peruano.

Un número bastante grande de datos, obtenidos durante muchos años. por barcos alemanes y diversas expediciones científicas, permitieron a Schott $(1931,1935)$ hacer la primera descripción científica de las corrientes del Pacífico Oriental. El denomina "región de las Galápagos" a la que nos ocupa y usa, por primera vez, el nombre de Corriente del Perú a esa masa de agua que se traslada de Sur a Norte. desde aproximadamente Antofagasta -es la opinión de Shott - hasta mas o menos los 5 US y luego toma dirección Oeste, pasando por el Sur de las Galápagos para incorporarse a la Corriente Sudecuatorial. El mismo autor se ocupa de la Corriente El Niño, de aguas tropicales, que aparece cada 2,7 o 10 años y cubre parte de las aguas frías antes citadas, determinando a la vez, lluvias inusuales en la costa septentrional del Perú.

Estudios posteriores (Wooster et al., 1958; Wooster \& GilMartin, 1961; Wyrtky, 1968) han permitido que hoy día tengamos una imagen mucho más clara del sistema de corrientes del Pacífico Oriental, tanto en sus regiones ecuatoriales, como en la mericional. Sabemos, por lo tanto, que existe un sistema ecuatorial constituído por las corrientes Norecuatorial y Surecuatorial, que corren paralelas, de Este a Oeste y entre las cuales se desarrolla una contracorriente ecuatorial. Este sistema tiene una posición latitudinal variable (entre 0 y $7^{\circ} \mathrm{N}$ ) y da lugar a lo que se denomina también "Zona de Convergencia Intertropical" (ZCIT - ITCZ).

Debajo de la región tropical nombrada, que llega con ciertas variables hast los $3^{\circ} 30^{\prime}$ (abundante pluviosidad, alta concentración de humedad y elevada temperatura), se sitúa el sistema de corrientes del Pacífico Sur, representado por la Corriente de Humboldt que, según acuerdos internacionales. debe denominarse Corriente Costera Peruana (CCP); por una corriente paralela situada mar adentro (Corriente Oceânica Peruana (COP); y por una contracorriente sub-superficial, situada entre ambas (o Corriente de Cromwell). Hacia los 4 a $7^{\circ} \mathrm{S}$, en situación variable, encontramos el frente oceánico en el cual se encuentran las aguas tropicales del Norte y las aguas frías de la Corriente Costera Peruana. Cuando dicho frente se diluye y la CCP pierde fuerza, se produce el denominado " $\mathrm{Fe}$ nómeno El Niño", que puede ser considerado como un factor perturbador, y se manifiesta por una invasión de aguas tropicales a la zona litoral del Perú, con una distribución que llega a veces a los 7 u $\gamma^{\circ} \mathrm{S}$ y otras veces, hasta los $120^{\circ} 13^{\circ} \mathrm{S}$ y al cual ya Schott (1935), como hemos visto, le da una periodicidad variable.

Tanto la CCP, como la Corriente Subsuperficial o Corriente de Cromwell, determinan el importante afloramiento de aguas, en la zona costera del Perú y del Norte de Chile, cuyas características son ampliamente conocidas.

Las regiones o provincias biogeográficas citadas por la mayor parte de los autores, para el Pacífico Sudoriental. han sido dos: la Panameña o Pacífico-panamena (ver p.ej. Olsson, 1961) y la Peruano-chilena o simplemente Peruana (p.ej. Marincovich, 1973). La primera, de características tropicales, abarcaría desde el Golfo de California hasta Tumbes $\left(3^{\circ} 30^{\prime} \mathrm{S}\right)$, en el extremo septentrional del Perú. La segunda se iniciaría en este último punto y se extendería hasta el límite meridional de la Corriente de Humboldt, quedando constituída por aguas más frías de lo que se esperaría para la latitud. Conviene destacar, respecto a la provincia peruano-chilena, que Lopez (1963) la divide en dos distritos, uno peruano, de $6^{\circ} 56^{\prime}$, hasta Coquimbo en Chile ( $\left.30^{\circ} \mathrm{S}\right)$; y otro centro-chileno, de Coquimbo a Valdivia $\left(40^{\circ} \mathrm{S}\right)$. Esta propuesta se basa en que hay una zona de mezcla con la fauna magallánica entre Valdivia y Valparaíso y luego, hacia el Norte, se va acentuando cada vez más la fauna septentrional.

En este trabajo proponemos que se considere, además de las dos regiones principales ya citadas, una tropical oceánica coincidente con la Corriente Oceánica Peruana y un distrito intermedio de transición, entre los 3 y $7^{\circ} \mathrm{S}$, donde se puede notar la influencia de aguas tropicales y de aguas frías. Esta opinión fue sustentada primeramente por Koepcke (1951) y retomada por el autor (1963 a y 1970). Los estudios ictiológicos (Hildebrand. 1946: Hildebrana \& Barton, 1949; Chirichigno, 1974), malacológicos (Keen, 1958; Olsson, 1961; Peña, 1970, 1971; Vegas, 1963b y 1968; Cantera \& Contreras, 1977) y carcionológicos (Del Solar et al., 1970), han confirmado la idea inicial.

\section{Las especies características}

Citar especies características, desde el punto de vista biogeográfico, es siempre un riesgo, por diversas razones. En primer lugar, porque ninguna provincia biogeográfica está totalmente encerrada y libre de invasiones faunísticas de provincias vecinas. En segundo lugar, porque rara vez se tiene a la mano una revision completa de la bibliograffa y es posible que aparezca algún registro de última hora o algún trabajo poco conocido o de poca circulación, que contradiga las afirmaciones de sunuesta caracterización. De todas maneras, vamos a ensayar la caracterización, utilizando los peces, mo luscos y crustáceos, que son los grupos zoológicos que más conocemos.

Peces

Aunque pudiera parecer más difícil, por la gran movilidad que tienen, la caracterización a base de este grupo zoológico es bastante clara. Es posible que ciertos fenómenos fisiológicos, relacionados principalmente con los intercambios osmóticos, determinen una exigencia mayor para estos organismos. Pero, en todo caso, podemos citar dos especies que nunca se apartan de la zona influenciada por la CCP: Engraulis ringens, la anchoveta y Odonthestes regia regia, Atherinidae, un pejerrey muy frecuente en esa zona. Ninguna de las dos especies llega al distrito Intermedio y, menos aún por cierto, a la provincia tropical septentrional.

Las otras especies que podemos citar para la provincia peruano-chilena, no demuestran una exigencia tan precisa. El bonito Sarda sarda chilensis es una especie que prefiere esa provincia, ya que en aguas tropicales existe Sarda velox orientalis. Otras especies que parecen preferir la provincia peruano-chilena, serían: Acanthistius pictus, Brevoortia maculata chilcae, Charcharinus porosus, Cheilodactylus variegatus, Chromis crusma, Dasyatis brevis, Emblemaria hudsoni, Etropus ectenes, Hemilutjanus macrophtalmus, Isacia conceptionis, Menticirrhus ophicephalus, M. paitensis, Merlu. ccius gayi, Mustelus mento, Ophichthus pacifici, Paralichthys adspersus, P. microps, Pinguilabrum punctatum, Psammobatis asper, Sardinops sagax sagax, Scartichthys xiphiodon, Sciaena callaensis, S. fasciata, S. gilberti, Sebastes chamaco, Seriolella porosa, Sicyases sanguineus, Syngnathus - independencia, Trachurus symmetricus murphyi. Todas ellas invaden la región intermedia en forma más - menos notable, pudiéndoseles fijar el límite septentrional hacia los $3^{\circ} 30^{\prime} \mathrm{S}$.

En relación con la fauna o distrito intermedio, la exclusividad específica es aún más difícil, ya que, como lo dijimos más arriba, existen invaciones meridionales v septentrionales frecuentes; o también, de la COP. Sin embargo, Chirichigno (1974) cita las s1guientes especies importantes, que no han sido registradas fuera 
de esta zona: Anthias sechurae, Dorsopsetta norma, Hemanthias delsolari, Hemicaranx sechurae, Muraena insularum, Mycteroperca olfax. Myxodagnus macrognathus, Ophicthus grandimaculatus, Otophidium fulvum, Peristedion crustosum, Pogonophis fossatus, Psammobatis aguja, Scorpaena afuerae, Spheroides andersonianus, Synchiropus talarae, Uroconger varidens. Ellas representan, junto con otras menos conocidas, el $4.31 \%$ de las especies citadas en la obra señalada, mientras el grupo anterior, de la Corriente de Humboldt o CCP está incluído en el $20.3 \%$ de las especies citadas.

Las especies de la provincia Pacífico-panameña ocupan, en cambio, el $46.3 \%$ de las citadas por Chirichigno (1974), conformando en consecuencia, el grupo más numeroso, en cuanto a repartición geográfica. Algo similar sucede con los moluscos, como veremos luego. Esto confirma la regla, generalmente aceptada, de que en aguas tropicales, la variedad de especies es mayor, pero las poblaciones específicas son más restringidas. Casi todas estas especies tienen una distribución que va del Golfo de California a Paita (Perú) o lugares cercanos (Zorritos, Máncora, Sechura, Bayóvar). Entre las especies mảs importantes citariamos: Abudefduf (varias especies), Anchoa (varias especies), Bagre (v. esp.), Brotula clarkae, Diplectrum pacificum, Echidna noctuma, Epinephelus (v. esp.), Hypoglossina stomata, H. tetrophthamus, Lutjanus (v. esp.), Menticirrhus nasus, $M$. undulatus, Muraenu lentiginosa, Mustelus dorsalis, Ophichtus triserialis, Pomadsys (v. esp.), Pristis zephyreus, Raja equatorialis, Spheroides angusticeps, S. trochocephalus, Stellifer (v. esp.), Syngnathus auliscus, Synodus marchenae, S. scituliceps, S. sechurae, Urotrygon (v. esp.)

Hay un grupo de especies (19.6\%) que son citadas por Chirichigno (1974), tanto para el distrito tropical como para el peruano chileno, unas veces hasta Chimbote $\left(9^{\circ} \mathrm{S}\right)$, otras hasta El Callao $\left(12^{\circ} \mathrm{S}\right)$ y otras hasta Pisco $\left(14^{\circ} \mathrm{S}\right)$. En este caso, tenemos un conflicto, porque el simple registro no nos indica si se trata de una presencia permanente, o si, como lo consideramos nosotros, es una presencia periódica o temporal, debida al Fenómeno El Niño. Es decir, que los cambios detemperatura y salinidad del agua, permiten a especies tropicales bajar hasta los $14^{\circ} \mathrm{S}$. en lugar de hacerlo como es normal, hasta los 6 o $7^{\circ} \mathrm{S}$. Ya Schweigger (1964) habia sugerido para el caso de Pisco que, de vez en cuando, se veían aparecer especies de aguas más cálidas. Citamos algunos ejemplos: Cetengraulis mysticetus, Cynoscion stolzmanni, Diapterus peruvianus, Elops saurus affinis, Epinephelus labriformis, Harengula peruana, Lutjanus guttatus, Menticirhus panamensis, Nematistius pectoralis, Ophichthus zopochir, Polynemus approximans, Rhynodon typus, Scomberomorus maculatus sierra, Synodus evermanni, Trachinotus rhodopus.

Podemos citar como casos dignos de estudio más profundo, los de los géneros Hirundichthys, Ophichthus, Prionotus, Scorpaena, Symphurus y Sphoeroides, pues parecen existir especies para cada distrito, cuya distribución habría que precisar. Igualmente, merece mayor atentión el género Mugil, sobre todo por las particularidades biológicas de cada espécie.

Por otro lado, tendríamos las especies tropicales de mar afue$\mathrm{ra}$, entre las cuales podemos citar, esencialmente, los géneros Exocoetus, Hirundichthys, Makaira, Thunnus y Xiphias. Todas las especies pueden acercarse a la costa, cuando hay invasiones de aguas calientes, desde el Norte o desde el Oeste.

Finalmente, tenemos las especies de distribución cosmopolita, como Alpias vulpinus, Anchoa spinifer, Coryphaena hippurus, Galeorhinus zyopterus, Hexanchus griseus, Lepidocybium flavobrunneum, Mugil cephalus, Mola mola, y los géneros Myxine, Prionace, Remora, Scomber, Sphyma.

\section{Moluscos}

Los estudios realizados sobre moluscos (Cantera \& Contreras, 1977; Keen, 1958; Olsson, 1961; Pena, 1970, 1971a y 1971b; Vegas, 1963b y 1968) señalan una situación similar para este grupo zoológico, en lo que se refiere a la distribución costera:

a) Los moluscos tropicales, con un rango geográfico de gran amplitud, entre el Golfo de California y las cercanías de Paita, conforman el grupo mayoritario, pasando seguramente el $50 \%$ de las especies descritas (en este caso es más difícil hacer una estadística más precisa, porque no hay una revisión global del grupo). Podemos citar las especies más frecuentes (Bivalvos): Anadara grandis, A. similis, A. tuberculosa, Atrina amura, Aequipecten tumbezensis, Donax asper, $D$, carinatus, $D$, obessus, Mactra califomica, Macoma columbensis, Mytella guyanensis, Ostrea columbiensis, O. megodon, O. prismatica, Pecten perulus, Pteria sterna, Protothaca asperrima, Tivela byronensis, T. argentina, Trachycardium senticosum. (Gasterópodos): Anachis dalli, A. rugulosa, Bulla punctulata, Cerithidea mazatlanica, C. pulchra, Calyptraea mamillaris, Crepidula incursa, C. excavata, C. onyx, Cypraea cervinetta, C. arabicula, Conus gladiator, C. ximenes, Malea ringens, Mitra leus, M. tristis, M. swainsoni, Littorina fasciata, $L$ varia, Nerita funiculata, Olivella collumellaris, $O$. volutella, Polinicies glauca, P. helicoides, P. uber, Pyrene major, P. strombiformis, Strombus peruvianus, Tegula byroniana, Thais biseria. lis, T. triangularis, T. especiosa, Turritella broderipana, Turbo magnificum.

b) Para la región intermedia $\left(3\right.$ a $\left.7^{\circ} \mathrm{S}\right)$, no parecen existir registros de Bivalvos ( $y$ aquí podría emprenderse un estudio ecológico interesante, para explicar esta situación), por razones que es dificil de adelantar. En cambio, entre los Gasterópodos, citaríamos: Cypraea nigropunctata, Fissurella asperella, F. latimargina. ta, $F$, microthema, Littorina paytensis, Pyrene paytensis, Olivella broggi. Aquí conviene citar el caso de moluscos que son tropicales, pero que no aparecen en regiones de manglares, probablemente por condiciones abióticas y particularmente sedimentarias. Es decir, en el caso del Perú, no han sido encontrados en Tumbes, pero hay registros de regiones tropicales más septentrionales que no señalan el biotopo, y registros de la zona límite de Máncora, Paita, a veces Pimentel (Bivalvos): Chione compta, $C$, subimbricata, Donax mancorensis, Petricula lenguafelis, $P i$ tar elenensis, Tivela hians, T. planulata; (Gasterópodos): Cerithium adustum, Conus purpurascens, Muricanthus oxyacantha, Natica broderipiana, N. grayi, Olivella gracilia, Sinum debile. Esta situación confirma la opınión de Cervigon (1978), segun la cual, las características físico-químicas de las aguas son el factor fundamental de distribución geográfica; pero, además, debe examinarse los factores ecológicos (en este caso, tipo de sedimentos, capacidad de filtración, quizá tactismos) y los factores específicos y evolutivos que determinan mayor o menor capacidad de adaptación.

c) En la provincia peruano-chilena, podemos citar las siguientes especies principales: Bursa ventricosa, Calyptraea trochiformis, Collisella orbignyi, C. viridula, Concholepas concholepas, Crepidula dilatata, C. onyx, Fissurella (v. esp.), Littorina peruviana, Monodonta nigerrima, Nassarius gayi, Oliva peruviana, Polinices helicoides, Risogaster niger, Sinum concavum, Siphonaria lessoni. Thais callaoensis, $T$, chocolata, $T$. delessertiana, Tegula atra; (Bivalvos): Aulacomya ater, Aequipecten purpuratus, Brachidon. tes purpuratus, Choromytilus chorus, Donax peruvianus, Glycy. meris maculata, G. ovata, Lithophaga peruviana, Mesodesma do nacum, Perumytilus purpuratus, Semimytilus algosus, Solen macha.

d) Existe un caso interesante, ya señalado antes, (Vegas, 1968) y que todavía no puede ser explicado con claridad. Algunos moluscos, que debemos clasificar de tropicales, interrumpen su distribución, como ya se dijo, entre los 4 y $7^{\circ} \mathrm{S}$, viniendo del Norte. Pero reaparecen en los alrededores del puerto de Pisco $\left(14^{\circ} \mathrm{S}\right)$, sin que se les haya encontrado en localidades intermedias. Se puede señalar, esencialmente, los Gasterópodos Cantharus fusiformis y Marginella curta y los Lamelibranquios Mexicardia procerum, Raeta undulata, Semele corrugata y Spondilus princeps. Esta situación podría ser explicable en peces, nadadores por excelencia y que, como ya lo señalamos, bajan en las épocas en que se produce el Fenómeno El Niño. Pero es difícil encontrar una respuesta, por ahora, para los Moluscos. No se puede tratar de substrato ni de régimen acuático, porque existen similares a todo lo largo de la costa peruana. Tendría que buscarse en los factores climáticos y paleogeográficos, una explicación más adecuada.

\section{Crustáceos}

El caso de los crustáceos (Chirichigno, 1970; Del Solar et al., 1970) pude ser agrupado em cuatro posibilidades, el de las aguas tropicales, el de las aguas intermedias $\left(3^{\circ} 30^{\prime}\right.$ a $\left.7-8^{\circ} \mathrm{S}\right)$, el de las aguas propias de la CCP y el grupo de amplia distribución.

Para las aguas tropicales, debemos citar el importante grupo de los Penaeidae (Penaeus vannamei, P. occidentalis, P. californensis, $P$. stylirostris y otras especies menos importantes); el de los cangrejos de manglares (Ucides occidentalis, Uca princeps, U. panamensis, $U$. maracoani, U. stylifera); algunos Portunidae (Portunus panamensis, $P$. iridiscens, $P$. acuminatus); las langostas Panulirus y el cangrejo araña Euphylax dovii. Este último es interesante, porque aparece como una plaga, nadando casi a flor de agua en grandes concentraciones y hasta 45 millas de la costa (DEL SOLAR, 1972), pudiendo servir como indicador del fenómeno "El Niño".

Para las aguas intermedias, sólo hemos encontrado dos especies que parecería que sólo existen allí, pero pienso que faltan mayores investigaciones al respecto. Se trata de Palaemon peruanus y Xantho crenatus. 
En la región bañada por la CCP o Corriente de Humboldt, citaríamos a Chryphiops caementarius, Callianassa incinata, diversos Paguridae (Paguristes y Pagurus), Munida gracilipes, varios Porcellanidae (Allopetrolisthes y Petrolisthes), los Albuneidae (Blepharipoda spinimana y Lepidopa chiliensis) y Ocypode gaudichaudii, que no pasa más al norte de los $5^{\circ} \mathrm{S}$, pero parece reaparecer en regiones más septentrionales.

El grupo más numeroso es el de crustáceos de amplia distribución, es decir, que vienen de la región tropical e invaden la provincia peruano-chilena; o que incluso siguen más al sur de ésta. Podríamos citar: Blepharipoda occidentalis, Emerita analoga, E. emerita, E. rathbunae; varios Calappidae (Hepatus chiliensis, H. kossmanni, Mussia gaudichaudii; los Cancridae Cancer polyodon y C. edwardsii; varias especies de Callinectes $(C$. toxotes y $C$. arcuatus, principalmente); algunos Xanthidae (Platyxanthus cokeri, $P$. crenulatus, $P$. orbignyi, Paraxanthus barbiger); cuatro e cinco especies de Pinnotheridae y algunas especies de la familia Grapsidae (Grapsus grapsus, Leptograpsus, Pachigrapsus). Es interesante señalar que $E$. analoga también desaparece hacia los $5^{\circ} \mathrm{S}$, pero parece reaparecer en latitudes más septentrionales.

\section{Conclusiones}

Los datos existentes hasta el presente, permiten mantener la proposición de considerar una zona costera intermedia entre las provincias pacífico-panameña y peruano-chilena y una zona tropical, correspondiente a la Corriente Oceánica Peruana.

Se debe intensificar los estudios de biogeografía ecológica, para aclarar los problemas discutidos en el presente trabajo y otros más que seguramente se harán patentes en un próximo futuro.

\section{Referencias bibliográficas}

CANTERA, J. \& CONTRERAS, R. 1977. Moluscos del Pacífico Colombiano. Sem. Océano Pacífico Sudamer., Cali (en prensa).

CERVIGON. F. 1978. Peces bentónicos del Atlántico septentrional y del Caribe. Sem. Latinoamer. Ecol. bentónica y sedumentación, Montevideo. (en prensa).

CHIRICHIGNO, N. 1970. Lista de crustáceos del Perú (Decapoda y Stomotopoda). Inf. IMARPE, (35), Lima

CHIRICHIGNO, N. 1974. Clave para identificar lus peces marinos del Perú. Inf. IMARPE, (44), Lima.
DEL SOLAR, E. 1972. Addenda al Catálogo de crustáceos del Perú. Inf. IMARPE, (38), 21p.

DEL SOLAR, E.; BLANCAS, F. \& MAYTA, R. 1970. Catálogo de crustáceos del Perú. Lima, Imp. Miranda.

HILDEBRAND, S 1946. A descriptive catalog of the shore fishes of Perú. Bull. Smiths. Inst., (189).

HILDEBRAND, S. \& BARTON, O. 1949 A collection of fishes from Talara, Perú. Smiths. Misc. Coll., $111(10)$

KEEN, M. 1958. Sea shells of tropical West America. Stanford Univ. Press.

KOEPCKE, H.W. \& M. 1951. División ecológica de la costa peruana. Ser. Div. Cient., (3), Div. Pesq. y Caza, Min. Agric., Lima.

LOPEZ, R. 1963. Problemas sobre la distribución geográfica de los peces marinos suramericanos. Museo Arg. Bernardino Rivadavia, Hidrobiologia, 1(3):111-135.

MARINCOVICH, L. 1973. Intertidal mollusks of Iquique, Chile. Nat. History Bull., Los Angeles County, Science Bull. $16.49 \mathrm{p}$.

OLSSON, A.A. 1961 Panamic Pacific Pelecypoda. Paleontol. Res. Inst., Ithaca.

PEÑA, M. 1970. Zonas de distribución de los gasterópodos marinos del Perú. Anales Cient. U.A. Lima, 8(3-4):153-170.

PEÑA, M. 1971a. Gasterópodos de los manglares del Perú. Anales Cient. U.A.Lima, 9(1-2):46-55.

PEÑA, M. 1971b. Zona de distribución de los bivalvos marinos del Perú. Anales Cient. U.A. Lima, 9(3-4):127-138.

SCHOTT, G. 1931. Der Peru-Strom. Annal. Hydrogr., (5-7).

SCHOTT, G. 1935. Geographie des Indischen und Stillen Ozeans. Hamburg. Verlag Boysen.

SCHWEIGGER, E. 1964. El litoral peruano. Lima, Impr. Miranda.

VEGAS-VELEZ, M. 1963a. La fauna, en Informe Nacional sobre las Zonas Aridas. Conf. Latinoamer. Reg. Aridas, Bs. As.

VEGAS-VELEZ, M. 1963b. La zona de Littorina en la costa peruaana. Anales Cient. U.A.Lima, I(2):174-193

VEGAS-VELEZ, M. 1968. Revisión taxonómica y zoogeográfica de algunos gasterópodos y lamelibranquios marinos del Perú Anales Cient. U.A. Lima, 6(1-2):1-29.

WOOSTER, W. \& CROMWELL, T. 1958. An oceanographic description of the Eastern tropical Pacific. Bull. Scripps Inst. Oceanogr., 7:169-282.

WOOSTER, W. \& GILMARTIN, M 1961. The Peru-Chile undercurrent. J. mar. Res., 19(3):97-122.

WYRTKY, K. 1968. Circulation and water masses in the eastern equatorial Pacific Ocean. Oceanol. Limnol., 1(2):117-147. 